\title{
Caracterización panorámica del español hablado en Colombia: fonología y gramática*
}

\author{
JULIO ALEXANDER BERNAL CHÁVEZ** \\ Instituto Caro y Cuervo, Colombia \\ julio.bernal@caroycuervo.gov.co \\ CAMILO ENRIQUE DÍAZ ROMERO \\ Instituto Caro y Cuervo, Colombia \\ camilo.diaz@caroycuervo.gov.co
}

Recepción: 29 de agosto de 2016

Aprobación: 31 de octubre de 2016

Forma de citar este artículo: Bernal Chávez, J.A., \& Díaz Romero, C.E. (2017). Caracterización panorámica del español hablado en Colombia: fonología y gramática. Cuadernos de Lingüística Hispánica, (29), 19-37. doi: http://dx.doi.org/10.19053/0121053X. n29.2017.5845

* Artículo de revisión elaborado en el marco del "Grupo de Investigación Lingüística del Instituto Caro y Cuervo", Colombia.

** Profesor del Instituto Caro y Cuervo, Colombia. Líder del "Grupo de Investigación Lingüística”. Candidato a Doctor en Lingüística de la Universidad de Bergen, Noruega. Código ORCID: 0000-0002-1652-0145

*** Profesor del Instituto Caro y Cuervo, Colombia. Miembro del "Grupo de Investigación Lingüística". Candidato a Doctor en Lingüística de la Universidad del País Vasco, España. Código ORCID: orcid.org/0000-0002-1811-7181 


\section{Resumen}

En este panorama se presentan las características más generales del español hablado en Colombia, en especial, en los niveles fonológico, morfológico y sintáctico. Se exponen, en primer lugar, una contextualización sobre los estudios del español que se usa en el país y las propiedades que son comunes a la mayoría de los hispanohablantes colombianos. En segundo lugar, siguiendo la división diatópica propuesta por Montes (1982), se hace mención de las particularidades lingüísticas de cada superdialecto. Por último, se muestran las cualidades del español hablado en zonas de contacto con las lenguas indígenas y se postulan algunas de las tareas pendientes de investigación sobre las diferentes variedades diatópicas y de contacto de la lengua oficial en el nivel nacional.

Palabras clave: Español hablado en Colombia, Superdialectos, Contacto españollenguas indígenas.

\section{Panoramic Characterization of Spanish Spoken in Colombia: Phonology and Grammar}

\section{Abstract}

This article presents a panorama of some of the most general characteristics of Colombian Spanish, especially at phonological, morphological and syntactic levels. We begin with a contextualization of existing studies about Spanish in Colombia, along with a characterization of the average Colombian Spanish speaker. Secondly, following the diatopical division stated by Montes (1982), a reference to the linguistic peculiarities of each superdialect is made. Finally, we present the features of the Spanish spoken in areas that have contact with indigenous languages, and pending matters for further research regarding the different contact and diatopic varieties of Spanish in Colombia.

Key words: Colombian Spanish, Superdialects, Contact between Spanish and Indigenous Languages. 


\section{Caractérisation panoramique de l'espagnol parlé en Colombie: phonologie et grammaire}

\section{Résumé}

Dans ce panorama, on présente les caractéristiques les plus générales de l'espagnol parlé en Colombie, en particulier, dans les niveaux phonologique, morphologique et syntactique. On expose, en premier lieu, une contextualisation sur les études de l'espagnol, qui est utilisé dans les pays, et les propriétés qui sont communes à la majorité des hispanophones colombiens. En deuxième lieu, suivant la division diatopique proposée par Montes (1982), on mentionne les particularités linguistiques de chaque superdialecte. En fin, on montre les qualités de l'espagnol parlé dans les zones de contact avec les langues autochtones et on postule quelques taches qui restent à accomplir concernant la recherche sur les différentes variétés diatopiques et de contact de la langue officielle au niveau national. autochtones.

Mots clés: Espagnol parlé en Colombie, Superdialectes, Contact espagnol-langues

\section{Caracterização panorâmica do espanhol falado na Colômbia: fonologia e gramática}

\section{Resumo}

Neste panorama apresentam-se as características mais gerais do espanhol falado na Colômbia, em especial, nos níveis fonológico, morfológico e sintático. Expõem-se, em primer lugar, uma contextualização sobre os estudos do espanhol usado no país e as propriedades que são comuns à maioria dos hispanofalantes colombianos. Em segundo lugar, seguindo a divisão diatópica proposta por Montes (1982), faz-se menção das particularidades linguísticas de cada superdialeto. Por último, mostram-se as qualidades do espanhol falado em zonas de contato com as línguas indígenas e postulam-se algumas das tarefas pendentes de pesquisa sobre as diferentes variedades diatópicas e de contato da língua oficial a nível nacional.

Palavras chave: Espanhol falado na Colômbia, Superdialetos, Contato espanhollínguas indígenas. 


\section{Contextualización}

Los estudios sobre el español hablado en Colombia tienen una tradición que surge con gramáticos y filólogos como Rufino José Cuervo, Miguel Antonio Caro, Ezequiel Uricoechea, Marco Fidel Suárez, entre otros. Lozano (2012a, p. 14) considera que, quienes, durante el siglo XIX, hicieron estudios acerca de la lengua como Apuntaciones críticas del lenguaje Bogotano (Cuervo, 1872), El castellano en mi tierra (Suárez, 1910) tenían el propósito de contrastar la norma lingüística culta con el léxico popular y regional.

Sería en 1942, con la creación del Instituto Caro y Cuervo (ICC), cuando se retoman los estudios de Cuervo y se desarrollan investigaciones lexicográficas y dialectológicas, viéndose fortalecido este tipo de estudios en el marco del macroproyecto de investigación conocido como Atlas Lingüístico-Etnográfico de Colombia (ALEC), para el que se inician labores de indagación en 1947 con la revisión de los atlas lingüísticos elaborados en Estados Unidos (Kurath, 1939-1943), así como la observación de los desarrollos europeos consignados en el Atlas Lingüístico de la Península Ibérica (Navarro, 1930-1954) y el Atlas Lingüístico de Francia (Gilléron \& Édmont, 1902-1910), lo que se continúa con una serie de estudios realizados durante más de dos décadas que culminan con la publicación en 1983 del último tomo del ALEC (Flórez, 1982-1983).

Esta investigación permitió llegar a un conocimiento científico de los dialectos del español hablado en Colombia, así como proporcionar isoglosas léxicas del país y de cientos de artículos que describen los rasgos lingüísticos de las más de 250 localidades a las que fueron los investigadores del proyecto.

Paralelamente, el equipo de lexicografía del ICC trabajó en el Diccionario de construcción y régimen de la lengua castellana, cuyo primer volumen publicó Cuervo (1886) en París, y cuya labor culminó en 1994. Este equipo también se incorpora al proyecto lexicográfico de Augsburgo Nuevo Diccionario de Americanismos con el Nuevo Diccionario de Colombianismos (Haensch \& Werner, 1993). En la actualidad se ha emprendido el desarrollo de un nuevo diccionario de colombianismos bajo la coordinación de María Clara Henríquez y Nancy Rozo: el Diccionario de Colombianismos-DiCol (Rozo et al., 2015).

El Departamento de Dialectología del ICC, después de culminar el ALEC, realiza estudios que profundizan en el español de Bogotá, los cuales culminan con la publicación 
del texto El español hablado en Bogotá: análisis previo de su estratificación social (Montes et al., 1998). El estudio de la norma lingüística culta de Bogotá que se adscribe al Proyecto de estudio coordinado de la norma lingüística culta de las principales ciudades de Iberoamérica y de la Península Ibérica, para el que se iniciaron encuestas en 1973 y cuya última publicación fue elaborada por Otálora (1997).

Actualmente, el equipo de investigadores de la línea de Lingüística de Corpus del ICC (LICC) adelanta la creación de un corpus oral digital del Instituto Caro y Cuervo en el que se incluyen las grabaciones realizadas en el ALEC, las del babla culta de Santafé de Bogotá y El español hablado en Bogotá. Además se trabaja en el macroproyecto Un ALEC para el Siglo XXI, en el cual se generará un Sistema de Información Geográfica (SIG) y un sitio de internet con una versión del ALEC en la que se podrán hacer consultas complejas y además de los mapas aparecerán 17.000 fotografías digitalizadas de las localidades visitadas y grabaciones de 190 localidades.

El ALEC dio espacio a múltiples trabajos investigativos respecto a las características dialectológicas perceptuales, como ocurre con el estudio de Bernal et al. (2014), fonológicas, como las reinterpretaciones de los dialectos hechas con teorías modernas como la Fonología Natural (Ramírez, 2015; Díaz, 2016), de relación entre las variedades diatópicas con variedades diastráticas en el español hablado en Colombia registradas en el estudio de Lozano (2012b), o el compilado de File-Muriel \& Orozco (2012), en el cual también se toma en consideración no solo variables fonológicas como los casos de debucalización y elisión de /s/ (Brown \& Brown, 2012), sino también las sintácticopragmáticas, como el uso de la cópula ser como focalizador (Méndez, 2012).

Como gran conclusión de los estudios lingüísticos acerca del español de Colombia, tenemos la propuesta de Montes de dos grandes superdialectos ${ }^{1}$ (1982, p. 30): la zona centro-septentrional (superdialecto A o andino), y el meridional (superdialecto B 0 costeño).

Mora et al. (2004) hacen una caracterización léxica de los dialectos del español de Colombia precisando las subdivisiones territoriales que se podían encontrar dentro de cada uno de los superdialectos a partir de la revisión del léxico recogido en los mapas del ALEC. En la Tabla 1 se presenta un resumen de la división dialectal del español de Colombia que se estableció en el estudio.

1 Según Mora et al. (2004, p. 13) el superdialecto es una división territorial de gran extensión bajo el criterio de rasgos lingüísticos, principalmente fonológicos y gramaticales, que son comunes a varios dialectos. 
Tabla 1. División dialectal del español hablado en Colombia a partir del ALEC

\begin{tabular}{|c|c|c|c|}
\hline SUPERDIALECTOS & DIALECTOS & SUBDIALECTOS & \begin{tabular}{|l|l|} 
LÉXICOS REGIONALES \\
\end{tabular} \\
\hline \multirow{7}{*}{ COSTEÑO } & \multirow{5}{*}{$\begin{array}{l}\text { COSTEÑO } \\
\text { ATLÁNTICO }\end{array}$} & Cartagenero & Bolívar \\
\hline & & Samario & Magdalena \\
\hline & & Guajiro & Guajira \\
\hline & & Atlántico interior & Córdoba \\
\hline & & & Sucre, Cesar \\
\hline & \multirow{2}{*}{$\begin{array}{l}\text { COSTEÑO } \\
\text { PACÍFICO }\end{array}$} & Pacífico norte & Chocó \\
\hline & & Pacífico sur & Cauca/Nariño \\
\hline \multirow{8}{*}{ ANDINO } & \multirow{3}{*}{$\begin{array}{l}\text { ANDINO } \\
\text { OCCIDENTAL }\end{array}$} & Antioqueño-caldense & Antioqueño - caldense \\
\hline & & Caucano-valluno & Valluno/ Caucano \\
\hline & & Andino sureño & Nariñense \\
\hline & \multirow{3}{*}{$\begin{array}{l}\text { ANDINO } \\
\text { ORIENTAL }\end{array}$} & Santandereano & $\begin{array}{l}\text { Nortesantandereano } \\
\text { Santandereano }\end{array}$ \\
\hline & & Cundiboyacense & $\begin{array}{l}\text { Cundinamarqués } \\
\text { Boyacense }\end{array}$ \\
\hline & & Tolimense-huilense & $\begin{array}{l}\text { Tolimense } \\
\text { Huilense }\end{array}$ \\
\hline & \multirow{2}{*}{ LLANERO } & Llanero norte & $\begin{array}{l}\text { Arauca, } \\
\text { Casanare }\end{array}$ \\
\hline & & Llanero sur & $\begin{array}{l}\text { Guaviare, } \\
\text { Caquetá }\end{array}$ \\
\hline
\end{tabular}

Fuente: Mora et al. (2004)

En la siguiente sección, se presentarán las características lingüísticas registradas en los superdialectos del español hablado en Colombia observados desde los estudios del ALEC a estudios más recientes.

\section{Características generales del español hablado en Colombia}

\subsection{Nivel fonológico}

Se reconoce la presencia de casos de elisión de oclusivas sonoras intervocálicas (Flórez, 1978, pp. 207, 237). Palabras como buevo y lado se producen como ['une. $\beta_{\mathrm{T}}$ ] ['uxe.o] y ['la.ðָo] ['la.o] respectivamente. Se identifican casos de sonorizaciones de /s/ en posición de ataque de sílaba (Vaquero, 2003a, p. 37). Por ejemplo, casa y sala se suelen expresar como ['ka.sa] y ['sa.la] ${ }^{2}$ respectivamente.

2 Se hace uso de símbolos del Alfabeto Fonético Internacional (IPA, 1999). La transcripción de los diptongos del español se hace siguiendo los criterios planteados por Hualde (2014), por los cuales los elementos vocálicos que introducen o siguen al máximo de sonoridad en la sílaba son marcados con el diacrítico de no silábico. 
Predomina la realización debucalizada del fonema fricativo velar, esto es, /x/ se realiza como [h] (RAE, 2011, p. 195; Hualde, 2014, pp. 149-150). Términos como ajo y gesto se manifiestan como ['a.ho] y ['hes.to] respectivamente.

La vocal /e/ se desnucleariza en contacto con vocales que no son palatales, lo que hace que surja un diptongo creciente (Flórez, 1963). Expresiones como leopardo y iqué bubo? se suelen pronunciar como [lion. 'par.ðָo] y ['kin $\beta_{\tau}$ o] respectivamente.

\subsection{Nivel morfológico}

Se conserva la oposición aspectual entre la forma de conjugación de participio y el pretérito simple siendo el primero valorado como aspecto imperfecto y el segundo, perfecto; atributos que son los diametralmente contrarios a los del español de la Península Ibérica (Aleza, 2010a, p. 146): ha trabajado o babía trabajado da cuenta de una actividad cuya finalización no se define, en tanto que trabajó expresa una acción de la cual su terminación es precisada.

El morfema diminutivo |-it- | presenta dos alomorfos: [ik], en forma escrita <-ic- >, que ocurre ante raíces que terminan con una sílaba portadora del fono [t], como ocurre en gatico [ga'tiko] y maletica [male'tika], e [it], en forma escrita <-it- $>$, que ocurre en los demás contextos, por ejemplo, pequeñito [peke' nito] (Vaquero, 2003b, p. 27; Areiza y Flórez, 2016, p. 87). En ocasiones, el diminutivo se emplea con fines afectivos o de cortesía (Aleza, 2010a, pp. 196-197): disculpe, ime podría regalar un tintico? (= deme un tinto). Se hace uso extendido del sufijo -azo con referencia a problemas, fracasos, percances y situaciones adversas que se presentan de manera repentina: "porrazo, trompazo, trastazo, leñazo, tortazo, pelotazo, piñazo, guamazo, toletazo” (RAE, 2009, p. 400).

El género gramatical lo puede definir el modificador. Ejemplos de ello son "el radio [...], el sartén [...], la piyama" (Aleza, 2010a, p. 220). Se identifica la presencia de |-ear| como un derivador verbal constante: "macanear [...], abalear [...], macanear [...], puertear [...], sabanear" (Enguita, 2010, p. 315).

\subsection{Nivel sintáctico}

El verbo pelear(se) puede emplearse como transitivo (Aleza, 2010a, p. 180). Por ejemplo, se suelen escuchar expresiones como peleo cada peso, ellas pelean voto a voto, ellos pelean la medalla de bronce.

Se pueden encontrar registros del verbo adberir(se) como no pronominal (Aleza, 2010a, p. 177). Se identifican casos del tipo yo adbiero al partido político de Pepe. Se identifica la presencia de la cópula ser con función de focalizador (Pato, 2010; Méndez, 
2012): trabajaba era por millones de pesos, me ponía era a cantar, nos reconocen mucho es por eso, me dirigí era a estudiar.

Se emplea la expresión dizque como contracción de dicen qué, cuya función es la de reportar información de la que no se presenta ningún grado de certeza (Aleza, 2010a, pp. 173-174): dizque Juan viene mañana, dizque aprobó el examen, dizque viene de la India. Además, esta contracción se ha lexicalizado al punto de que puede funcionar como adverbio, marcador de evidencialidad, sustantivo y adjetivo, según el contexto sintáctico en que se presente (Bernal, 2009).

El dequeísmo se emplea constantemente tanto en el habla culta como el habla popular (Flórez, 1964, p. 15): opino de que el campeón de la liga debería ser un equipo de Santander, piensa de que lo importante es ganar la curul de senador. Se identifican registros de la perífrasis irá a + infinitivo con sentido de conjetura (Aleza, 2010a, p. 171): irá a trabajar en la escuela (= iría a trabajar en la escuela).

Se conservan usos de la perífrasis venir a infinitivo con el valor de inicio de una acción (Aleza, 2010a, p. 163): vengo a escribir un poema (= iré/voy a escribir un poema). La preposición hasta se emplea como referencia del momento de inicio de una acción (DeMello, 1992): abren basta las cinco de la mañana (= abren desde/a partir de las cinco de la mañana).

Se registran casos de uso del clítico se como marcador que opera en oraciones de concordancia refleja con plural (Vaquero, 2003b, p. 29): se venden casas, se arreglan zapatos, se reparan motores.

Se usa la locución a como dé lugar como equivalente de cueste lo que cueste (Aleza, 2010a, p. 211): no se rindan, hagan la tarea a como dé lugar, firmen el tratado a como dé lugar. Casos similares son los de al frente en lugar de enfrente (Aleza, 2010a, p. 212) y por (si) las dudas en el sentido de por si acaso (Aleza, 2010a, p. 215).

Son locuciones de uso frecuente: a mano limpia; por debajo de cuerda (con el sentido de hacer algo de manera oculta); de pronto (como equivalente de posiblemente); de apuchos (en pequeñas cantidades); de un pelo (Aleza, 2010a, pp. 215-219). Se registran casos de pluralización del pronombre clítico lo cuando hay un objeto indirecto: di el libro a tus padres, se los di (Montes, 1992).

Los hispanohablantes colombianos tienen actitudes consolidadas de la división del español hablado en Colombia entre los superdialectos andino y costeño (Bernal et al., 
2014; Bernal, 2016), lo que hace importante presentar, desde la lingüística, cuáles son los rasgos característicos de estas variedades diatópicas.

\section{Propiedades características del superdialecto costeño}

\subsection{Nivel fonológico}

Se observan registros de debucalización /s/ en el contexto de coda de sílaba interna de palabra: está esperando [eh. 'ta.eh.pe. 'rãn.do], dos bombres ['do.hõm.bre] (Flórez, 1963, p. 272; Espejo, 2016, p. 101).

El fonema /s/ tiende a elidirse en contexto de final de palabra fonológica: mujeres [mu. 'he.re], la de atrás [la.dia' tra]. También se registran casos de ensordecimiento de la consonante que sigue después de la /s/ debucalizada, lo que conduce a que expresiones como los vasos se pronuncien como [loh.' fa.so] o [loh. 'фa.so] (Montes, 1982, pp. 3132). Por hipercorrección, a menudo se registran casos de refuerzo con fricativa alveolar final en nombres y apellidos: Aracelys, Nerys (Montes, 1982, p. 37).

En ocasiones, también se encuentran casos de asimilación de /s/ y / $/$ / en contexto preconsonántico, en especial, cuando precede a oclusivas, conduciendo a la creación de una secuencia consonántica geminada: bosque ['bok.ke], estera ['et.te.ra], cobarde [ko. 'ß̧ą.de] (Montes, 1974; De Granda, 1977; Becerra, 1980; 1985; Páez, 2009). El fonema vibrante simple /r/ se elide en contexto final de palabra (Flórez, 1963, p. 273; Montes, 1982, p. 38): mujer [mu.' he], hacer [a.'se], gozar [go.'sa]. Esto conduce a que se elimine la marca tradicional segmental de infinitivo.

Se documentan registros de la alternancia de // / / (Montes, 1982, p. 39; Lipski, 1996): los soldados [lo.sor. 'da.o], sal [sar], árbol ['al.bol], personal [pel.so. 'nal]. En algunas situaciones, se obtienen casos de vocalización de /r/ en contexto preconsonántico y final de palabra, lo que conduce a crear diptongos decrecientes y triptongos: puerco [' puei. ko], amor [a. 'moi] (Montes, 1982, p. 39; 1992, p. 524).

La articulación de /n/ es velar en contexto final de sílaba (Flórez, 1963, p. 272; Canfield, 1981, p. 7; Montes, 1982, p. 45): jamón [ha. 'mõy], fin [fĩ̄]. Se han observado nasalizaciones de vocales contiguas y no contiguas a una nasal, haya o no desaparecido la consonante nasal, y en circunstancias muy diversas (Aleza, 2010b, p. 90).

Según Montes $(1982 ; 1996)$, el fonema africado postalveolar se realiza con una fase de disolución fricativa breve o con una disolución palatalizada. Al respecto, la RAE (2011) enuncia que "en el dialecto costeño de Colombia, la africada sorda se realiza como [ $\left.\mathrm{t}^{\mathrm{s}}\right]$, con predominio del momento oclusivo y un momento fricativo poco perceptible que a veces 
suena como una t palatalizada [tj]" (p. 208). En poblaciones como Iscuandé, Nariño, se registra una realización alveolar de la africada (Montes, 1974, p. 411).

El yeísmo $(/ \mathrm{j} /, / K />/ \mathrm{j} /)$ es un fenómeno sonoro frecuente que se puede encontrar tanto en la costa pacífica como en el Caribe, con tendencias a presentar realizaciones debilitadas del fonema fricativo palatal -yuca ['ju.ka]- (Montes, 1996; Peña, 2015, pp. 190-191).

Respecto de las oclusivas sonoras, hay tendencia a su elisión en contexto intervocálico: chubasco [tfu. 'as.ko] (Vaquero, 2003a, p. 27). También, se registran casos de pérdida de oclusiva alveolar sonora /d/ en posición final de palabra: pinté la pared [pĩn. 'te.la.pa. 're] (Orozco, 2009, p. 97).

Se documentan casos de pronunciación de palabras con el grafo $<\mathrm{h}>$ con el fono fricativo faríngeo [h] o glotal [h] (Flórez, 1960, p. 178; RAE, 2011, p. 188): la palabra baya la pueden pronunciar como ['ha.ja] o ['ha.ja].

Algunas palabras que son diptongos las articulan como hiatos: "cri-ollo, ca-úcho, cri-ar, cri-ado, rumi-ar, bri-oso" (Montes, 1982, p. 19).

\subsection{Nivel morfológico}

Como tal, predomina el tuteo como fórmula de tratamiento de confianza e igualdad. Solo en ciertas zonas de la costa del Pacífico, las que son más cercanas con la zona andina, se encuentran casos de voseo (Montes, 1982, p. 42).

El verbo hacer se conjuga con número plural ante términos referentes al tiempo, que son valorados como sujetos gramaticales: hacen días, meses, años, etc. (Montes, 1982, p. 42). Se usa femenino en palabras como azúcar, liendre, fantasma, calor (Flórez, 1949, p. 135; Montes, 1959, pp. 83-84).

Predominan los compuestos de raíz nominal sin terminación en /i/ seguido de una raíz adjetival: barbamarilla, bocabierta, cabezadura, etc. (Montes, 1977, pp. 657-658).

\subsection{Nivel sintáctico}

Se identifican registros de construcciones sintácticas conformadas por el auxiliar ser en subjuntivo combinado con un verbo en participio (Montes, 1976a): si el oro fuera valido más boy, yo fuera sido más rico.

En regiones donde la población que predomina es afrodescendiente, tanto en el Caribe (De Friedemann \& Patiño, 1983) como en el Pacífico (Huer \& Ruiz, 1998), se 
encuentran construcciones de doble negación, esto es, la producción de dos marcas del adverbio no dentro de una misma oración: ino me molestes no!, no trabajo boy no. También se identifica el copiado de clíticos de objeto indirecto dentro de una misma oración con una forma en infinitivo (Orozco, 2009, p. 99; Orozco \& Díaz-Campos, 2016): se lo iba a entregárselo, yo le quería darle las flores.

Hay casos de discordancia entre el auxiliar haber, que se conjuga en tercera persona singular, y el pronombre sujeto expresado en primera persona del singular (Orozco, 2009, p. 99): yo le ha mostrado el nuevo billete de 20 barras (20.000 pesos colombianos).

\section{Propiedades características del superdialecto andino}

\subsection{Nivel fonológico}

El fonema /s/ se conserva en final de sílaba, salvo en el habla informal de Bogotá, en donde se registran unos esporádicos casos de debucalización (Montes, 1996). La articulación del fonema /s/ presenta un continuum de coronalidad en la realización fonética, que va desde la realización ápico-alveolar o retrofleja en Antioquia, Caldas, Quindío y Risaralda hasta la realización lámino-alveolar de Santander, el Altiplano Cundiboyacense, Cauca y Valle del Cauca. También se reconoce la presencia de variación en la fonación de /s/ en contexto intervocálico (Flórez, 1978; Montes 1996, p. 40; Ramírez, 2015), por lo que la pronunciación de una palabra como casas puede ser registrada en este superdialecto como ['ka.șas], ['ka.s్sas] [ ['ka.șaș], ['ka.șas] ], ['ka.șas] o ['ka.şas]].

Se identifican casos de posteriorización e incluso debucalización de la consonante fricativa labiodental sorda /f/ (Flórez, 1963, p. 268; RAE, 2011, p. 189): palabras como café, afán y famosa se dicen [ka.' he], [a.' han] y [ha. 'mo.sa] respectivamente.

Respecto de los fonemas róticos $(/ \mathrm{r} / \mathrm{y} / \mathrm{r} /)$, se reportan casos de asibilación, los cuales son frecuentes en departamentos como Nariño y Boyacá (Flórez, 1963, p. 271; Albor, 1971, p. 526): puerco y marrano suelen decirse como ['puez.ko] y [ma.'za.no] respectivamente.

Se destaca el mantenimiento del fonema aproximante lateral palatal $/ K /$ en palabras que portan el grafo <ll>: llave y fallo se pronuncian como [' $K \mathrm{a} . \beta_{\mathrm{r}}$ ] y [' fa.Ko] respectivamente (Flórez, 1978; Moreno de Alba, 1988; Lipski, 1996; Orduz, 2013).

En ocasiones, se registra la realización de los fonemas oclusivos sonoros como fonos oclusivos (Aleza, 2010b, p. 72), por lo que se pueden encontrar algunos casos de palabras como cabo y ave con la pronunciación correspondiente de ['ka.bo] y ['a.be]. En relación 
con el fonema africado palatal sordo $/ \mathrm{t} / \mathrm{s}$, se reconoce la presencia de un "sonido que suena como una consonante t palatal" (Montes, 1996, p. 136), esto es [t $\mathrm{t}]$.

\subsection{Nivel morfológico}

Se presentan dos tipos de fórmulas de tratamiento que están ausentes en el superdialecto costeño: la primera es el voseo, que posee su propio modelo de conjugación verbal -vos tenés, vos pensás, vos decís, etc.- Su extensión territorial va desde el departamento de Antioquia hasta el norte de Nariño, en el suroccidente del país (Flórez, 1953; Montes, 1967). La segunda es el pronombre sumercé, que es una variante histórica del pronombre usted (que proviene de vuestra merced), pero con posesivo en tercera persona singular (su merced). Este pronombre es empleado por hablantes de español en el altiplano cundiboyacense (Flórez, 1963, pp. 277-278).

Los compuestos de raíz nominal suelen ser con terminación en /i/ seguido de una raíz adjetival: barbiamarilla, boquiabierta, lengüilargo, etc. (Montes, 1977, pp. 657-658).

\subsection{Nivel sintáctico}

Existen casos de oraciones con la ausencia de los clíticos de objeto directo, en especial, en zonas andinas occidentales (Arboleda, 2000, p. 96; Zagona, 2002, p. 69). Por ejemplo, $\mathrm{X}$ dice: itrajiste la tarea? Z responde: no traje (= no lo traje), haga la tarea (=hágame la tarea).

Como se sabe, en Colombia se hablan, aparte del español, cerca de 68 lenguas indígenas, así como 2 lenguas criollas (Landaburu, 2004-2005, pp. 3-8). A continuación, se presentan algunas características que se han identificado en el español hablado en este país como resultado del contacto con algunas lenguas aborígenes.

\section{Características del español hablado en Colombia en situacio- nes de contacto con las lenguas indígenas}

\subsection{Nivel fonológico}

En el español hablado en las situaciones de contacto con el embera chamí, se documentan casos de interferencia por los cuales palabras que se pronuncian con el fono fricativo labiodental [f] en español, como foca o faro, son producidos con el fono oclusivo bilabial sordo aspirado $\left[\mathrm{p}^{\mathrm{h}}\right]$, que es la realización fonética del fonema $/ \mathrm{p}^{\mathrm{h}} /$ del embera chamí (Bedoya \& Restrepo, 1998, p. 6; Romero et al., 2008, p. 102). También hay vacilaciones en la fonación de la producción de sonidos obstruyentes en el español de los Chamíes, por 
ejemplo, se confunde "jarra con garra [...] se escribe dierra, en vez de tierra" (Romero et al., 2008, p. 102).

En zonas de contacto del español con lenguas como el yukpa, el ika, el tikuna, el piapoco y el sikuani, se registran casos de elisión de la fricativa alveolar sorda en contexto final de palabra (Rodríguez Cadena, 2008; Aleza, 2010b, p. 67).

En zonas de contacto del español con lenguas como el ika, el yukpa y el kogui, se documentan casos de elisión del fonema vibrante simple (Rodríguez Cadena, 2008; Aleza, 2010b, p. 78).

En zonas de contacto entre el español y las lenguas piapoco y sikuani se documenta que hay despalatalización de palabras con el fonema nasal palatal $/ \mathrm{n} /$ en español (Reinoso Galindo, 1999): "niño se pronuncia [nínio]" (Aleza, 2010b, p. 87). Hay casos de propagación de la nasalidad a morfemas enteros en palabras del español producidas por indígenas de la Sierra Nevada de Santa Marta, en especial, los Wiwas, comunidad que habla damana (Rodríguez Cadena, 2008, p. 139; Aleza, 2010b, p. 90), por lo que palabras como mesita pueden producirse como [mẽ. 'sĩ.tã].

Se registran casos de cierre de vocales en el español hablado por indígenas kamsá (Ávila Mora, 2001, p. 136; Aleza, 2010b, p. 89): palabras como caso, meta y solo son pronunciados como ['ka.su], ['mi.ta] y ['su.lu] respectivamente.

\subsection{Nivel sintáctico}

Se registran casos de discordancias de género en el español hablado en las situaciones de contacto con las lenguas sikuani, wayú y tikuna (Ramírez, 2009, p. 174; 2012, p. 674): la dato, esa padre, primero pregunta, la contralor, nuestro cortina, etc. También existen las discordancias de número por hablantes bilingües de español con las lenguas sikuani, wayú y tikuna (Ramírez, 2009, p. 175; 2012, p. 675): un pesos, él son bijo, un trabajos, ellos estudia, nosotros trabajo en la casa.

En el caso de los bilingües español-wayú, se registran casos de elisión de artículos y de cópulas en los registros que hacen de sus producciones en español (Ramírez, 2012, pp. 676-677): casa pequeño (= la casa es pequeña), niña enfermo (= la niña está enferma), gatos grande (=los gatos son grandes), etc.

También se documentan casos de construcciones frasales de doble posesivo en el español de zonas en contacto con el inga, esto es, la presencia, en una misma frase, del adjetivo y el pronombre posesivo (De Granda, 1997, p. 145): mi casa de mí, tu mascota de ti, etc. 


\section{Tareas pendientes de investigación}

En cuanto a zonas de contacto, el español hablado en zonas como el Vaupés o el Caquetá, regiones en donde se identifica la presencia de una amplia variedad de lenguas indígenas de familias indígenas como el Nadahup (Epps, 2008) o el Tukano-Occidental (Barnes, 1999), aún no ha sido tratado en ningún nivel de análisis lingüístico. También está pendiente de estudios el español hablado en las islas de San Andrés y Providencia, territorio conocido por el uso de una lengua criolla, así como por la presencia del uso del inglés en la cotidianidad (Davis, 2011; Moya, 2014).

Respecto de los estudios dialectológicos de la lengua española hablada en Colombia, la zona de los llanos orientales requiere de un estudio más profundo. En los tiempos de las grabaciones de ALEC, se hicieron solo registros de municipios del Meta como Restrepo y Puerto López (Lozano \& Montes, 1999, p. 614) y solo se produjo un esbozo exploratorio del habla de Casanare y Arauca en un estudio de Montes (1976b). También lo requiere el habla de los hispanohablantes no indígenas que viven en zonas como la región Amazónica, de los cuales, salvo los registros llevados a cabo en Leticia por Alvar (1977), no se encuentra nada que se haya hecho en los últimos 35 años.

\section{Referencias bibliográficas}

Albor, H. (1971). Observaciones sobre la fonología del español hablado en Nariño. Thesaurus, 26, 515-533.

Aleza, M. (2010a). Fonética y Fonología. Parte segunda: pronunciación. En M. Aleza \& J. Enguita (coords.) (2010). La lengua española en América: normas y usos actuales. Valencia: Universidad de Valencia.

Aleza, M. (2010b). Morfología y sintaxis. Observaciones gramaticales de interés en el español de América. Parte segunda: fenómenos gramaticales. En M. Aleza \& J. Enguita (coords.) (2010). La lengua española en América: normas y usos actuales. Valencia: Universidad de Valencia.

Alvar, M. (1977). Leticia: estudios lingüísticos sobre la amazonía colombiana. Con una monografía etnográfica de Elena Alvar. Bogotá: Instituto Caro y Cuervo.

Arboleda, R. (2000). El español andino. Forma y función, 13, 85-100.

Areiza Londoño, R., \& Flórez Ospina, M. (2016). Variantes del español colombiano y su efecto en la enseñanza del español como lengua extranjera. Marco Sociolingüístico. Cuadernos de Lingüística Hispánica, (27), 79-107. Doi: http://dx.doi.org/10.19053/0121053X.4211 
Ávila Mora, M. (2001). Descripción de la situación sociolingüística de la comunidad indígena kamëntsá de Sibundoy, Putumayo. Realización y transcripción del corpus hablado por bilingües. (Trabajo de grado). Universidad Nacional de Colombia, Bogotá, Colombia.

Barnes, J. (1999). Tucano. En R. M. W. Dixon \& A. Y. Aikhenvald (eds.). The Amazonian languages. Cambridge: Cambridge University Press.

Becerra, S. (1980). Consonantes implosivas en el español urbano de Cartagena de Indias (Colombia). En: G. Scavnicky (ed.). Dialectología hispanoamericana, estudios actuales. Washington, D.C.: Georgetown University Press.

Becerra, S. (1985). Fonología de las consonantes implosivas en el español urbano de Cartagena de Indias (Colombia): ensayo socio-lingüístico. Bogotá: Instituto Caro y Cuervo.

Bedoya, 0., \& Restrepo, M. (1998). Interferencia Lingüística entre la lengua epera y el español bablado en el Chocó. Pereira: Supercopias.

Bernal, J. (2009). Cambio lingüístico y lexicalización de dizque en Colombia. Hojas y hablas, 6, 53-60.

Bernal, J. (2016). Actitudes lingüísticas de los bogotanos hacia los dialectos del español hablado en Colombia y las variedades nacionales de esta lengua en los demás países de Hispanoamérica. (Tesis de doctorado). Universidad de Bergen, Bergen, Noruega.

Bernal, J., Munévar, A., \& Barajas, C. (2014). Actitudes lingüísticas en Colombia. En: A. B. Chiquito y M. Quesada (eds.). Actitudes lingüísticas de los hispanohablantes hacia el idioma español y sus variantes. Bergen: Universidad de Bergen.

Brown, E., \& Brown, E. L. (2012). Syllable-final and Syllable-initial/s/ reduction in Cali, Colombia: One variable or two? En R. File-Muriel y R. Orozco (eds.). Colombian Varieties of Spanish. Madrid y Frankfurt: Iberoamericana-Vervuert.

Canfield, D. L. (1981). Spanish Pronunciation in the Americas. Chicago: University of Chicago Press.

Cuervo, R. J. (1872). Apuntaciones críticas sobre el lenguaje bogotano, con frecuente referencia al de los países de Hispano-América. Bogotá: A. M. Guarín.

Cuervo, R. J. (1886). Diccionario de construcción y régimen de la lengua castellana. Tomo primero: $A-B$. París: A. Roger y F. Chernoviz, libreros editores.

Davis, C. (2011). Análisis sociolingüístico del conflicto entre el español, el inglés y la lengua nativa en San Andrés Isla. (Trabajo de grado). Universidad Tecnológica de Pereira, Pereira, Colombia. 
De Friedemann, N., \& Patiño, C. (1983). Lengua y sociedad en el Palenque de San Basilio. Bogotá: Instituto Caro y Cuervo.

De Granda, G. (1977). Estudios sobre un área dialectal hispanoamericana de población negra: las tierras bajas occidentales de Colombia. Bogotá: Instituto Caro y Cuervo.

De Granda, G. (1997). Replanteamiento de un tema controvertido. Génesis y retención del doble posesivo en el español andino. Revista de Filología Española, 77 (1/2), 139-147.

DeMello, G. (1992). Hasta = no hasta $/$ hasta no $=$ hasta, en el español hablado de once ciudades. Anuario de Letras, 30, 5-28.

Díaz, C. (2016). Phonological processes: Illustrations from velar vowels in Colombian Varieties of Spanish. En B. Ayhan (ed.). LILA '16 III. International Linguistics and Language Studies Conference. Estambul: DAKAM Publishing.

Enguita, J. (2010). Léxico y formación de palabras. En M. Aleza y J. Enguita (coords.). La lengua española en América: normas y usos actuales (pp. 261-316). Valencia: Universidad de Valencia.

Epps, P. (2008). A Grammar of Hup. Berlín: Mouton de Gruyter.

Espejo, M. (2016). Alofonía de /s/ en Colombia. Lingüística y Literatura, 69, 99-117.

File-Muriel, R., \& Orozco, R. (eds.) (2012). Colombian Varieties of Spanish. Madrid y Frankfurt: Iberoamericana-Vervuert.

Flórez, L. (1949). Cuestiones del español hablado en Montería y Sincelejo. Thesaurus, 5, 122-162.

Flórez, L. (1960). Pronunciación del español en Bolívar (Colombia). Thesaurus, 15, 174-179.

Flórez, L. (1963). El español hablado en Colombia y su atlas linguístico. Thesaurus, 18, 268-356.

Flórez, L. (1964). El español hablado en Colombia. Presente y futuro de la lengua española I, 5-77.

Flórez, L. (1978). Sobre algunas formas de pronunciar muchos colombianos el español. Thesaurus 33, 197-246.

Flórez, L. (dir.) (1982-1983). Atlas Lingüístico-Etnográfico de Colombia. Bogotá: Instituto Caro y Cuervo.

Gilléron, J., \& Édmont, É. (1902-1910). Atlas linguistique de la France. París: Champion.

Haensch, G., \& Werner, R. (dirs.) (1993). Nuevo diccionario de colombianismos. Tomo I del Nuevo diccionario de americanismos. Bogotá: Instituto Caro y Cuervo. 
Hualde, J. (2014). Los sonidos del español [con Sonia Colina]. Cambridge: Cambridge University Press.

Huer, K., y Ruiz, M. (1998). La doble negación chocoana: evidencia lingüística de un criollo anterior hablado por los esclavos del Chocó. (Tesis de maestría). Instituto Caro y Cuervo, Bogotá, Colombia.

International Phonetic Association-IPA (1999). Handbook of the International Phonetic Association. Cambridge: Cambridge University Press.

Kurath, H. (dir.) (1939-1943). Linguistic Atlas of New England, 3 vols, Providence: Brown University.

Landaburu, J. (2004-2005). Las lenguas indígenas de Colombia: presentación y estado del arte. Amerindia, 29/30, 3-22.

Lipski, J. (1996). El español de América. Madrid: Cátedra.

Lozano, M. (2012a). Breves notas sobre la investigación lingüística en Colombia. Cuadernos de Lingüística Hispánica, (19), 13-22. Recuperado de http://revistas.uptc.edu.co/revistas/ index.php/linguistica_hispanica/article/view/450/450

Lozano, M. (2012b). Diversidad regional y diversidad sociolingüística en el español de Colombia. En C. Patiño (ed.). El lenguaje en Colombia. Tomo I: Realidad Lingüística de Colombia. Bogotá: Instituto Caro y Cuervo.

Lozano, M., \& Montes, J. (1999). El Departamento de Dialectología. Thesaurus, 54, 529-657.

Méndez, C. (2012). On the syntax of the Focalizing Ser ('to be') structure in the Spanish of Bucaramanga. En R. File-Muriel y R. Orozco (eds.). Colombian Varieties of Spanish. Madrid y Frankfurt: Iberoamericana-Vervuert.

Montes, J. (1959). Del español hablado en Bolívar, Colombia. Thesaurus, 14, 82-110.

Montes, J. (1974). El habla del Chocó: notas breves. Thesaurus, 29, 409-428.

Montes, J. (1976a). Un arcaísmo gramatical en Colombia: la construcción del pretérito compuesto de subjuntivo con ser. Thesaurus, 31, 561-562.

Montes, J. (1976b). El español hablado en los Llanos Orientales (Primer Seminario sobre Investigaciones Antropológicas en los Llanos. Universidad Tecnológica de los Llanos Orientales, Villavicencio, junio). Bogotá: Instituto Caro y Cuervo.

Montes, J. (1977). Un tipo de composición nominal y el español atlántico. Thesaurus, 32, 653-659.

Montes, J. (1982). El español de Colombia: Propuesta de clasificación dialectal. Thesaurus, 37 , 23-92. 
Montes, J. (1992). El español hablado en Colombia. En C. Hernández (coord.). Historia y presente del español de América (pp. 519-542). Valladolid: Pabecal, Junta de Castilla y León.

Montes, J. (1996). Colombia. En M. Alvar (dir.). Manual de dialectología hispánica. Tomo 2. El español de América (pp. 134-145). Barcelona, Ariel.

Montes, J. et al. (1998). El español hablado en Bogotá: análisis previo de su estratificación social. Bogotá: Instituto Caro y Cuervo.

Mora, S. et al. (2004). Caracterización léxica de los dialectos del español de Colombia según el ALEC. Bogotá: Instituto Caro y Cuervo.

Moreno de Alba, J. (1988). El español en América. México: Fondo de la Cultura Económica.

Moya, D. (2014). La situación sociolingüística de la lengua creole de San Andrés Isla: el caso de San Luis. Colombian Applied Linguistics Journal, 16(1), 55-66.

Navarro, T. (dir.) (1930-1954). Atlas Lingüístico de la Península Ibérica, Madrid: CSIC.

Orduz, Y. (2013). La pérdida de distinción fonológica /j/ - / / / en el habla bogotana. Folios (segunda época), 37, 51-62.

Orozco, R. (2009). El castellano del Caribe colombiano a comienzos del siglo XXI. Revista Internacional de Lingüística Iberoamericana (RILI), 7(2), 95-113.

Orozco, R., \& Díaz-Campos, M. (2016). Dialectos del español de América: Colombia y Venezuela. En J. Gutiérrez-Rexach (ed.). Enciclopedia de Lingüística Hispánica. Nueva York: Routledge.

Otálora, H. (1997). Léxico del habla culta de Santafé de Bogotá. Bogotá: Instituto Caro y Cuervo.

Páez, D. (2009). Análisis instrumental de las consonantes oclusivas posnucleares seguidas de consonante en el dialecto del español del Chocó. (Trabajo de grado). Universidad Nacional de Colombia, Bogotá, Colombia.

Pato, E. (2010). El verbo "ser" focalizador en el español de Colombia”. Español Actual: Revista de español vivo, 93, 153-174.

Peña, J. (2015). Yeísmo en el español de América. Algunos apuntes sobre su extensión. Revista de Filología, 33, 175-199.

Ramírez, H. (2009). Interferencia y contacto de lenguas: español en fronteras bilingües de Colombia. Bogotá: Instituto Caro y Cuervo.

Ramírez, H. (2012). El español en territorios indígenas. En C. Patiño (ed.). El lenguaje en Colombia. Tomo I: Realidad Lingüística de Colombia. Bogotá: Instituto Caro y Cuervo. 
Ramírez, M. (2015). De la [s], [s $\left.{ }^{\mathrm{h}}\right],[\mathrm{h}],[\mathrm{P}], \varnothing$, a la [z], [z]: continuo de variación de /s/ en posición de coda silábica en el español de Colombia. En J. Santos (ed.). Armonía y contrastes: estudios sobre variación dialectal, histórica y sociolingüistica del español. Lugo: Axac.

Real Academia Española-RAE (2009). Nueva gramática de la lengua española. Vol. I, Morfología y Sintaxis I. Madrid: Espasa Libros, S. L.

Real Academia Española-RAE (2011). Nueva gramática de la lengua española. Fonética y Fonología. Barcelona: Espasa Libros, S. L. U.

Reinoso Galindo, A. (1999). Pedagogía y didáctica en el marco del programa de Licenciatura en Educación Básica para maestros piapoco, sicuani y criollos del Medio Guaviare. En L. D. Aguirre (comp.). Culturas, lenguas, educación, Barranquilla: Universidad del Atlántico.

Rodríguez Cadena, Y. (2008). Colombia. En A. Palacios (coord.). El español en América. Contactos lingüísticos en Hispanoamérica. Barcelona: Ariel.

Romero, F. et al. (2008). La enseñanza de español como segunda lengua. Un estudio de caso con niños nasa y embera-chamí. Pereira: Colciencias/Universidad Tecnológica de Pereira/ Consejo Regional Indígena del Cauca.

Rozo, N. et al. (2015). Diccionario de Colombianismos (DiCol), primera etapa. Disponible en: https://prezi.com/jqwpjcjhee4d/diccionario-de-colombianismos-dicol

Suárez, M. (1910). El castellano en mi tierra. Bogotá: Imprenta Eléctrica.

Vaquero, M. (2003a). El español de América. 1. Pronunciación. Madrid: Arco Libros, S. L.

Vaquero, M. (2003b). El español de América. 2. Morfosintaxis y Léxico. Madrid: Arco Libros, S. L.

Zagona, K. (2002). The Syntax of Spanish. Cambridge: Cambridge University Press. 